\title{
SREBP-1c, regulated by the insulin and AMPK signaling pathways, plays a role in nonalcoholic fatty liver disease
}

\author{
MOTOYUKI KOHJIMA ${ }^{1}$, NOBITO HIGUCHI ${ }^{1}$, MASAKI KATO ${ }^{1}$, KAZUHIRO KOTOH ${ }^{1}$, \\ TSUYOSHI YOSHIMOTO ${ }^{2}$, TATSUYA FUJINO ${ }^{2}$, MASAYOSHI YADA ${ }^{1}$, \\ RYOKO YADA ${ }^{2}$, NAOHIKO HARADA ${ }^{2}$, MUNECHIKA ENJOJI ${ }^{1}$, \\ RYOICHI TAKAYANAGI ${ }^{1}$ and MAKOTO NAKAMUTA ${ }^{2}$
}

\author{
${ }^{1}$ Department of Medicine and Bioregulatory Science, Graduate School of Medical Sciences, Kyushu University, Fukuoka; \\ ${ }^{2}$ Department of Gastroenterology, Kyushu Medical Center, National Hospital Organization, Fukuoka, Japan
}

Received October 16, 2007; Accepted December 21, 2007

\begin{abstract}
Nonalcoholic fatty liver disease (NAFLD) is a common liver disease whose prevalence has increased markedly. We reported previously that fatty acid synthesis was enhanced in NAFLD with the accumulation of fatty acids. To clarify the disorder, we evaluated the expression of genes regulating fatty acid synthesis by real-time PCR using samples from NAFLD $(n=22)$ and normal liver (control; $\mathrm{n}=10$ ). A major regulator of fatty acids synthesis is sterol regulatory element-binding protein-1c (SREBP-1c). Its expression was significantly higher in NAFLD, nearly 5 -fold greater than the controls. SREBP-1c is positively regulated by insulin signaling pathways, including insulin receptor substrate (IRS)-1 and -2. In NAFLD, IRS-1 expression was enhanced and correlated positively with SREBP-1c expression. In contrast, IRS-2 expression decreased by $50 \%$ and was not correlated with SREBP-1c. Forkhead box protein A2 (Foxa2) is a positive regulator of fatty acid oxidation and is itself negatively regulated by IRSs. Foxa2 expression increased in NAFLD and showed a negative correlation with IRS-2, but not with IRS-1, expression. It is known that SREBP-1c is negatively regulated by AMP-
\end{abstract}

Correspondence to: Dr Munechika Enjoji, Department of Medicine and Bioregulatory Science, Graduate School of Medical Sciences, Kyushu University, 3-1-1 Maidashi, Higashi-ku, Fukuoka 812-8582, Japan

E-mail: enjoji@intmed3.med.kyushu-u.ac.jp

Abbreviations: NAFLD, nonalcoholic fatty liver disease; NASH, nonalcoholic steatohepatitis; ACC, acetyl-CoA carboxylase; FAS, fatty acid synthase; SREBP-1c, sterol regulatory element-binding protein-1c; AMPK, AMP-activated protein kinase; IRS, insulin receptor substrate; Foxa2, forkhead box protein A2

Key words: nonalcoholic fatty liver disease, fatty acid, sterol regulatory element-binding protein-1c, AMP-activated protein kinase, insulin receptor substrate, forkhead box protein A2 activated protein kinase (AMPK) but expression levels of AMPK in NAFLD were almost equal to those of the controls. These data indicate that, in NAFLD, insulin signaling via IRS-1 causes the up-regulation of SREBP1-c, leading to the increased synthesis of fatty acids by the hepatocytes; negative feedback regulation via AMPK does not occur and the activation of Foxa2, following a decrease of IRS-2, upregulates fatty acid oxidation.

\section{Introduction}

Nonalcoholic fatty liver disease (NAFLD) is one of the most frequent causes of liver dysfunction (1-3) and its prevalence has been increasing markedly (4-6). Furthermore, nonalcoholic steatohepatitis (NASH), a severe form of NAFLD accompanied by hepatitis and fibrosis, may progress to cirrhosis and hepatic failure $(7,8)$. NAFLD is often found in patients with obesity and/or insulin resistance, however, its precise cause remains unclear. Therefore, it is important to understand the features of lipid metabolism, particularly fatty acid metabolism, in NAFLD. Previously, we evaluated the expression levels of genes involved in fatty acid metabolism in the liver with NAFLD and found that the expression of those genes including acetyl-CoA carboxylase (ACC) and fatty acid synthase (FAS), was up-regulated, indicating that fatty acid synthesis was enhanced in hepatocytes, leading to the accumulation of fatty acids $(9,10)$.

Sterol regulatory element-binding proteins (SREBPs) are membrane-bound transcription factors that regulate the expression of genes involved in lipid synthesis, and SREBP-1c positively regulates the expression of genes encoding lipogenic enzymes including ACC and FAS $(11,12)$. Insulin is a well-known stimulator of lipogenesis and activates the hepatic expression of SREBP-1c $(13,14)$. Insulin receptor substrate (IRS) proteins, a family of docking molecules, connect insulin receptor activation to essential downstream cascades, and the two major isoforms, IRS-1 and IRS-2, are highly expressed in the liver (15). In addition to the positive regulation of SREBP-1c expression, IRSs suppress fatty acid oxidation through the inhibition of a transcription factor, forkhead box protein A2 (Foxa2) (16). 
AMP-activated protein kinase (AMPK) is an energy sensor that regulates cellular metabolism including lipid metabolism (17). AMPK is activated by increased levels of cellular AMP, a marker of cellular energy stores (18). Activated AMPK stimulates ATP-producing catabolic pathways, such as fatty acid oxidation, and inhibits ATPconsuming processes, such as lipogenesis. Therefore, AMPK activation suppresses the expression of ACC and FAS via down-regulation of SREBP-1c $(19,20)$.

The mechanisms involved in the pathogenesis of NAFLD have not been thoroughly investigated, particularly in humans. Furthermore, little is known about the regulation of fatty acid synthesis in NAFLD. In this study, we evaluated the expression of lipogenesis regulator genes, SREBP-1c, IRS-1, IRS-2, Foxa2, and AMPK, in NAFLD.

\section{Patients and methods}

Tissue samples were obtained by liver biopsy from 22 patients with histologically diagnosed NAFLD, including 4 patients with NASH, who were admitted to the Kyushu University Hospital from 2004 to 2006. As controls, normal liver tissue was obtained by liver biopsy from 10 patients whose liver function tests and histological findings were completely normal. Written consent was obtained from the patients for this investigation. Real-time RT-PCR was performed as reported previously (10). Total RNA was prepared with TRIzol reagent (Invitrogen, Carlsbad, CA, USA) and cDNA was synthesized from $1.0 \mu \mathrm{g}$ RNA with GeneAmp $^{\text {TM }}$ RNA PCR (Applied Biosystems, Branchburg, NJ, USA) using random hexamers. Real-time RT-PCR was performed using LightCycler-FastStart DNA Master SYBR Green 1 (Roche, Tokyo, Japan) according to the manufacturer's instructions. The reaction mixture $(20 \mu \mathrm{l})$ contained LightCycler-FastStart DNA Master SYBR Green 1, 4 mM $\mathrm{MgCl}_{2}, 0.5 \mu \mathrm{M}$ of the upstream and downstream PCR primers, and $2 \mu \mathrm{l}$ of the first strand cDNA as a template. The target genes and primers used are shown in Table I. To control for variation in the reactions, all PCRs were normalized against $\beta$-actin expression. All results were shown as the mean \pm SD. Comparisons were made by the Mann-Whitney U test. For the correlation analysis between two continuous variables, a simple regression model was used.

\section{Results}

Expression of SREBP-1c, FAS, IRS-1, IRS-2 and AMPK. SREBP-1c is a transcription factor which positively regulates fatty acid synthesis-related genes such as FAS. In NAFLD, SREBP-1c expression was positively correlated with FAS expression $(\mathrm{r}=0.48, \mathrm{p}=0.03)$ and expressed at almost 5-fold higher levels in NAFLD than in the normal liver $(482 \pm 123 \%$, $\mathrm{p}=0.04$ ) (Figs. 1 and 2). SREBP-1c is positively regulated by insulin signaling pathways including IRSs and negatively regulated by AMPK. In NAFLD, IRS-1 expression was significantly higher than the controls $(170 \pm 21 \%, \mathrm{p}=0.03)$, whereas IRS-2 expression was lower $(72 \pm 23 \%, \mathrm{p}=0.34)$, and AMPK expression levels were roughly the same $(134 \pm 24 \%$, $\mathrm{p}=0.34)($ Fig. 2).
Table I. Primers used for analysis of the expression of fatty acid metabolism-related genes.

\begin{tabular}{llcrc}
\hline Gene & $5^{\prime}$ & $\begin{array}{l}\text { Forward primer } \\
\text { Reverse primer }\end{array}$ & $3^{\prime}$ & 3enBank $^{\mathrm{TM}}$ \\
& $5^{\prime}$ & accession no. \\
\hline SREBP-1c & \multicolumn{2}{c}{ GCGGAGCCATGGATTGCAC } & NM-004176 \\
& CTCTTCCTTGATACCAGGCCC & \\
AMPK & CAGGCATATGGTGGTCCATAGAG & AF100763 \\
& \multicolumn{3}{c}{ TCATGGGATCCACCTGCAGC } &
\end{tabular}

IRS-1 ACTTGAGCTACGGTGACGTG

NM-005544 GCGGAACTCATCACTCATGG

IRS-2 ACAACAACCACAGCGTGCGC GTAGAGGGCGATCAGGTACTTG

FAS

AGCTGCCAGAGTCGGAGAAC

NM-003749 TGTAGCCCACGAGTGTCTCG

NM-004104

Foxa2 CGGGCTCCATGAACATGTCG

NM-021784 GCGAGATGTACGAGTAGGGC

B-actin GCAAGAGAGGCATCCTCACC

NM-001101 CGTAGATGGGCACAGTGTGG

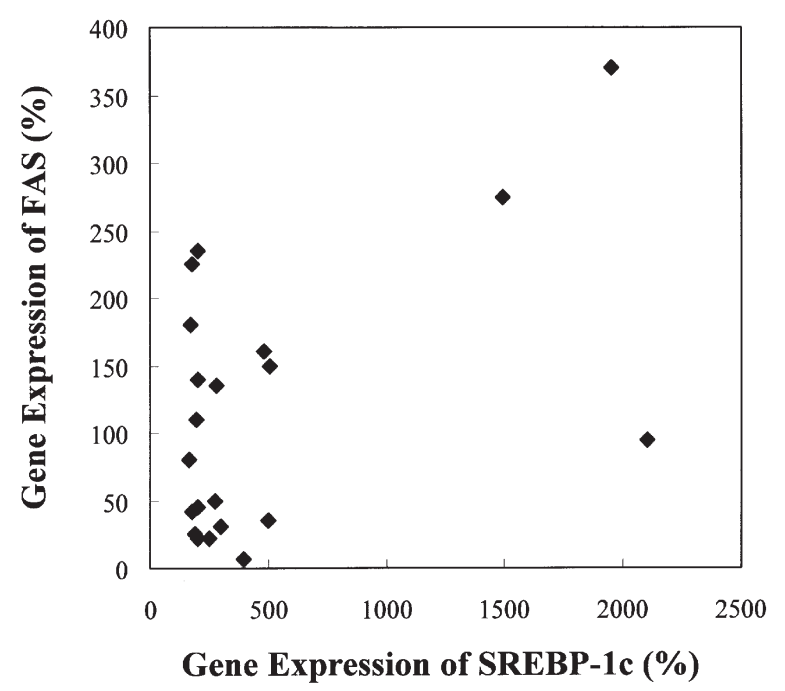

Figure 1. Correlation between FAS and SREBP-1c gene expression. There was a significant positive correlation between FAS and SREBP-1c expression. FAS, fatty acid synthase; SREBP-1c, sterol regulatory elementbinding protein-1c.

Relationship between SREBP-1c and IRSs or AMPK expression. In NAFLD patients, IRS-1 expression correlated positively with SREBP-1c expression $(\mathrm{r}=0.46, \mathrm{p}=0.03)$ (Fig. 3A), but IRS-2 expression did not $(r=-0.16, p=0.46)$ (Fig. 3B). There was no relationship between IRS-1 and IRS-2 expression ( $\mathrm{r}=-0.17, \mathrm{p}=0.32$ ) (Fig. 3C). AMPK expression did not show a significant correlation with SREBP-1c expression ( $\mathrm{r}=0.003, \mathrm{p}=0.99$ ) (Fig. 3D). IRSs recently have been reported to negatively regulate another transcriptional factor, Foxa2, which enhances fatty acid oxidation. Foxa2 expression was not significantly higher than the controls 

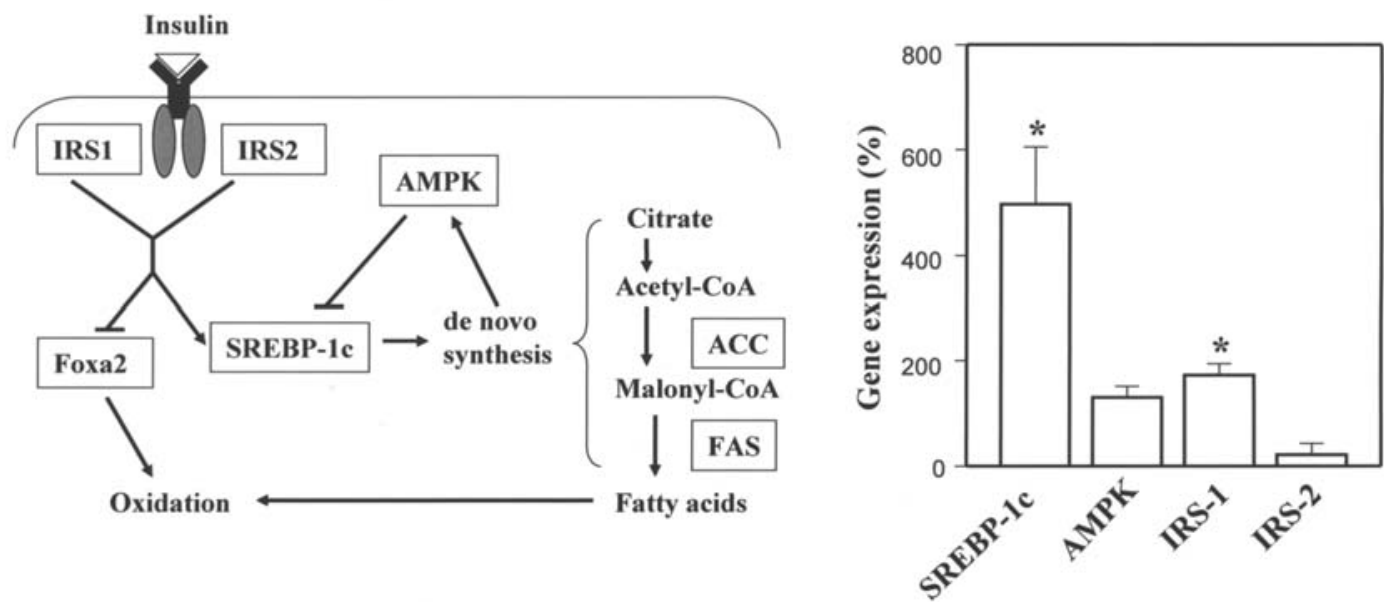

Figure 2. Real time RT-PCR analysis of the gene expression of SREBP-1c, IRS-1, IRS-2, and AMPK in NAFLD. SREBP-1c is positively regulated by insulin, and negatively regulated by AMPK. SREBP-1c and IRS-1 expression were significantly increased in NAFLD. *Statistically significant differences $(\mathrm{p}<0.05)$ compared to normal liver. ACC, acetyl-CoA carboxylase; FAS, fatty acid synthase; SREBP-1c, sterol regulatory element-binding protein-1c; AMPK, AMP-activated protein kinase; IRS, insulin receptor substrate; Foxa2, forkhead box protein A2.

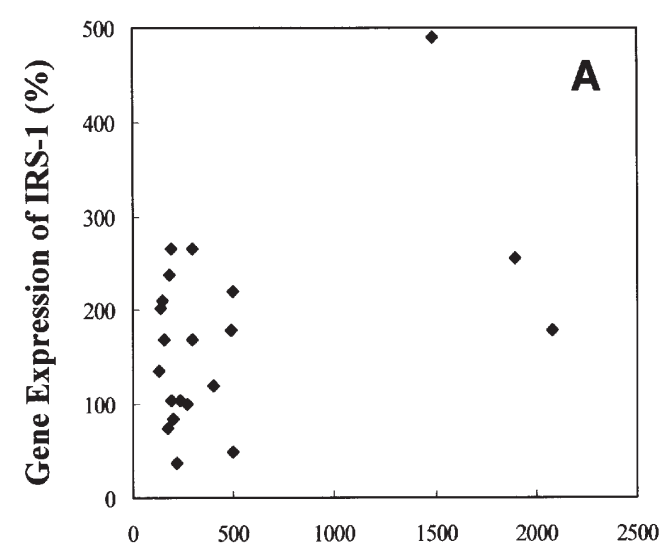

Gene Expression of SREBP-1c (\%)

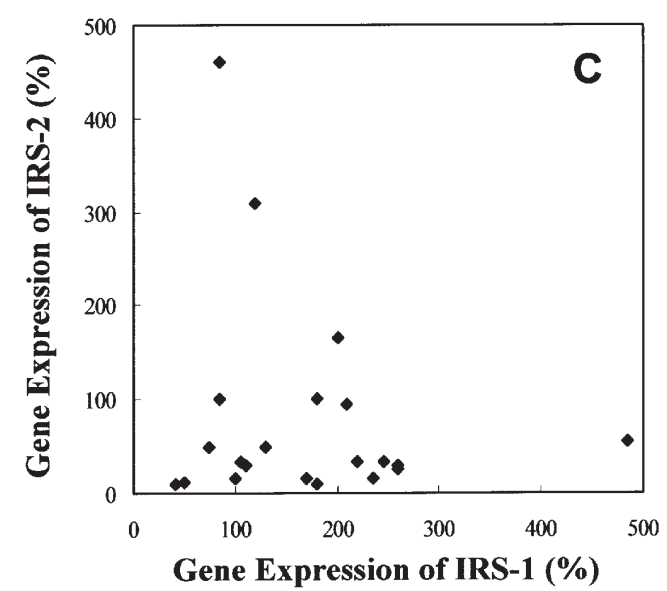

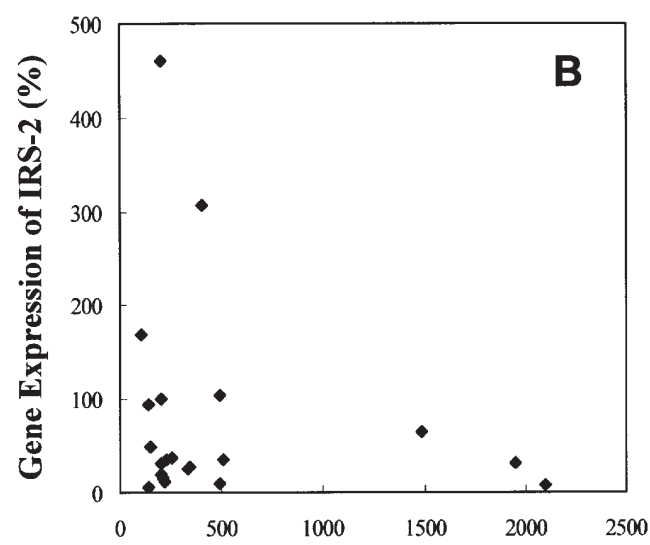

Gene Expression of SREBP-1c (\%)

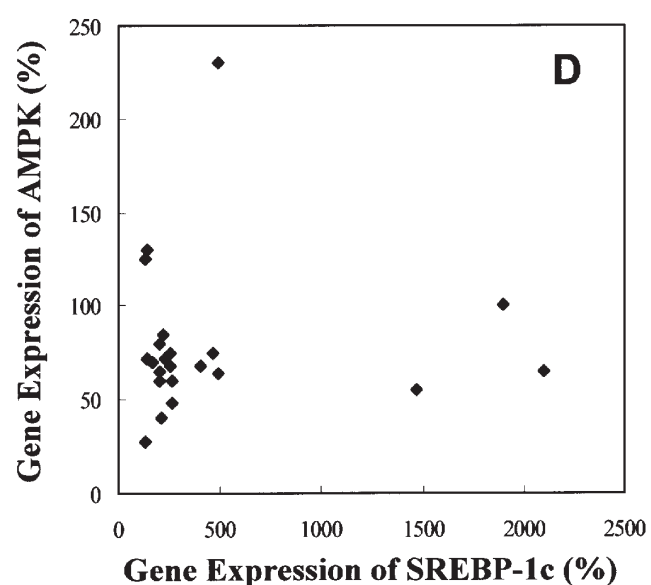

Figure 3. Correlation between IRS-1 and SREBP-1c (A), IRS-2 and SREBP-1c (B), IRS-1 and IRS-2 (C), and AMPK and SREBP-1c (D) in NAFLD. There was a significant positive correlation between IRS-1 and SREBP-1c expression. SREBP-1c, sterol regulatory element-binding protein-1c; AMPK, AMPactivated protein kinase; IRS, insulin receptor substrate.

$(148 \pm 25 \%, \mathrm{p}=0.24)$ but showed a negative correlation with IRS-2 expression ( $\mathrm{r}=-0.42, \mathrm{p}=0.04)$ (Fig. 4A). There was no correlation between Foxa2 and IRS-1 expression ( $\mathrm{r}=-0.04$, $\mathrm{r}=0.87$ ) (Fig. 4B).

\section{Discussion}

Previously we evaluated the expression of genes associated with fatty acid metabolism in NAFLD, and we found that 


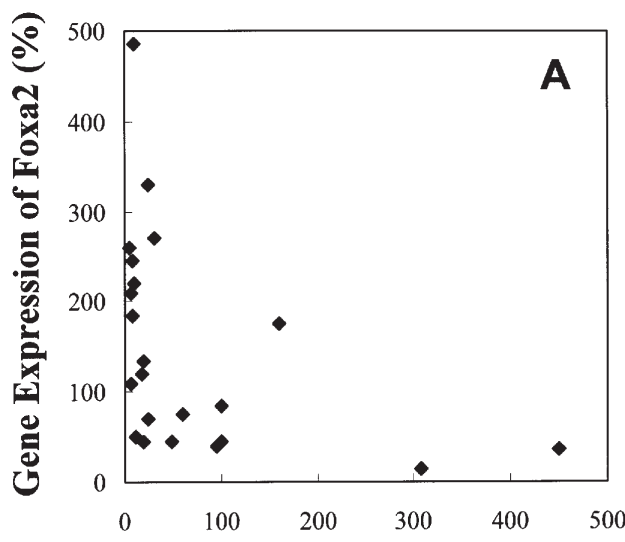

Gene Expression of IRS-2 (\%)

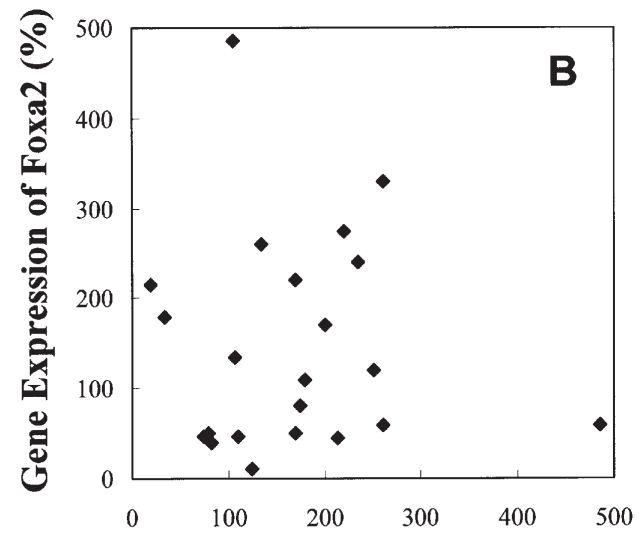

Gene Expression of IRS-1 (\%)

Figure 4. Correlation between IRS-2 and Foxa2 (A), and IRS-1 and Foxa2 (B) gene expression in NAFLD. There was a significant negative correlation between IRS-2 and Foxa2 expression.

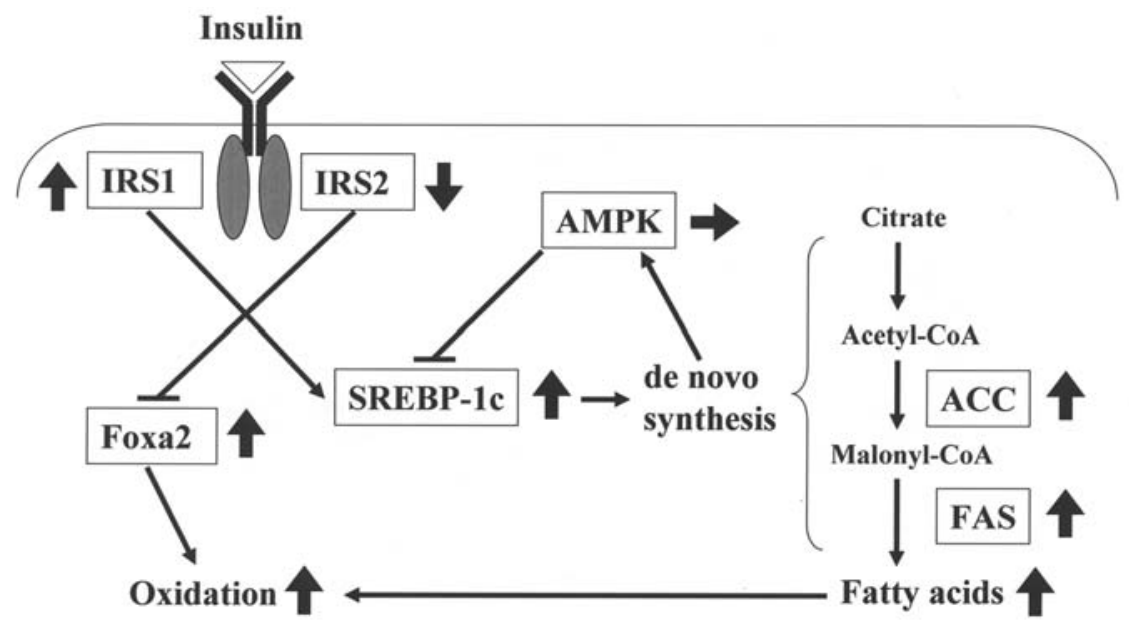

Figure 5. Summary of the present analysis of the expression of fatty acid synthesis and oxidation regulatory genes in NAFLD.

increased de novo fatty acid synthesis led to the increased accumulation of fatty acids in hepatocytes $(9,10)$. In the process of fatty acid synthesis, ACC converts acetyl-CoA, an essential substrate of fatty acids, to malonyl-CoA. FAS utilizes both acetyl-CoA and malonyl-CoA to form palmitic acid (C16:0). Both FAS and ACC expression are positively regulated by SREBP-1c, a transcription factor. In this study, a positive correlation was shown between SREBP-1c and FAS (Fig. 1) or ACC1 expression (data not shown) in NAFLD patients. These data indicate that one of the primary abnormalities of fatty acid metabolism in NAFLD is the impaired regulation of fatty acid synthesis by SREBP-1c.

SREBP-1c is positively regulated by insulin signaling pathways in which IRS-1 and -2 are major mediators. Interestingly, IRS-1 expression was enhanced in NAFLD and showed a positive correlation with SREBP-1c expression (Figs. 2 and 3A). In contrast, IRS-2 expression was reduced, although not significantly, and was not correlated with SREBP-1c expression (Figs. 2 and 3B). These results indicate that the insulin signaling pathways are disrupted in NAFLD, particularly in terms of the normally coordinated regulation between IRS-1 and -2. It is well known that
NAFLD is often accompanied by insulin resistance (1). When we define insulin resistance as dysregulation of the insulin signaling pathways, including IRSs, the decrease of IRS-2 expression might be pertinent to insulin resistance, although IRS-1 expression was rather increased. It was reported recently that hepatic IRS- 1 and -2 have complementary roles in the control of hepatocyte metabolism; IRS- 1 is linked more closely to glucose homeostasis and IRS-2 to lipid metabolism (21). Specifically, the selective knockdown of hepatic IRS-1 resulted in the up-regulation of glucogenic enzyme gene expression, whereas the knockdown of IRS-2 resulted in the up-regulation of SREBP-1c and FAS expression. It has also been reported that SREBP-1c suppresses IRS-2-mediated insulin signaling via direct interference with the IRS-2 promoter (22). These lines of evidence, including our own, indicate that up-regulated SREBP-1c not only enhances FAS expression but also suppresses IRS-2 expression via a negative feedback mechanism. In contrast, in our study, IRS-1 expression was enhanced and correlated with SREBP-1c expression. These results suggest that enhanced IRS-1 expression may be compensated by the suppression of IRS-2 expression, and that the switch from 
IRS-2- to IRS-1-dependent regulation may cause enhanced fatty acid synthesis by SREBP-1c in NAFLD. Further study is needed to clarify the mechanism of up-regulation of IRS-1 in liver with NAFLD.

IRSs also regulate fatty acid oxidation through transcription factor Foxa2 (16). Both IRS-1 and IRS-2 suppressed Foxa2, which positively regulates fatty acid oxidation. In our study, Foxa2 expression was slightly increased, which might be attributable to the net balance between decreased IRS-2 and increased IRS-1. Taken together with our finding that Foxa2 expression correlated to IRS-2 expression, IRS-2 might be a major regulator of Foxa2 in the liver with NAFLD. We reported previously that fatty acid oxidation was up-regulated in NAFLD although expression of PPAR $\alpha$, an activator of fatty acid oxidation, was significantly decreased (10). The results of this study suggest that the up-regulation of Foxa2 leads to an increase in fatty acid oxidation in NAFLD.

It has been proposed that AMPK acts as a metabolic master switch and its activation leads to a concomitant inhibition of energy-consuming biosynthetic pathways, such as fatty acid synthesis. AMPK activation inhibits ACC activity directly by phosphorylation (23), and inhibits ACC expression indirectly via the suppression of SREBP-1c (19). Our present and previous results show that AMPK expression was unchanged or, rather, increased and that SREBP-1c and ACC expression increased in parallel, indicating that the negative feedback regulation of SREBP-1c via AMPK failed in NAFLD. Recent findings showed that AMPK is activated by adiponectin (24) and that serum adiponectin levels were reduced in NAFLD (25). In our study, NAFLD patients showed lower serum adiponectin levels than the controls (data not shown); therefore, hypoadiponectinemia might contribute to unchanged AMPK expression in NAFLD.

In summary, our results in human NAFLD indicate the following (Fig. 5): i) up-regulated SREBP-1c contributes to the increased de novo synthesis of fatty acids, even in hepatocytes with fatty acid accumulation, ii) up-regulated IRS-1 is associated with the up-regulation of SREBP-1c, iii) suppression of SREBP-1c by AMPK failed in NAFLD and iv) activation of Foxa2 by decreased expression of IRS-2 upregulates fatty acid oxidation. Recently, it was reported that liver $\mathrm{X}$ receptor $\alpha(\mathrm{LXR} \alpha)$ plays an important role in lipogenesis through the activation of SREBP-1c (26). We are now investigating the relationship between $\operatorname{LXR} \alpha$ and SREBP-1c expression.

\section{Acknowledgements}

This work was supported in part by the research fund of the Clinical Research Foundation (Japan) and the Institute of Kampo Medicine (Japan).

\section{References}

1. Browning JD and Horton JD: Molecular mediators of hepatic steatosis and liver injury. J Clin Invest 114: 147-152, 2004.

2. Clark JM, Brancati FL and Diehl AM: The prevalence and etiology of elevated aminotransferase levels in the United States. Am J Gastroenterol 98: 960-967, 2003.
3. Angulo P: Nonalcoholic fatty liver disease. N Engl J Med 346: 1221-1231, 2002.

4. Nomura H, Kashiwagi S, Hayashi J, et al: Prevalence of fatty liver in a general population in Okinawa, Japan. Jpn J Med 27: 142-149, 1988.

5. Hilden M, Christoffersen P, Juhl E and Dalgaard JB: Liver histology in a 'normal' population - examination of 503 consecutive fatal traffic casualties. Scand J Gastroenterol 12: 593-597, 1977.

6. Bellentani S, Saccoccio G, Masutti F, et al: Prevalence of and risk factors for hepatic steatosis in northern Italy. Ann Intern Med 132: 112-117, 2000.

7. Ludwig J, Viggiano TR, McGill DB and Oh BJ: Nonalcoholic steatohepatitis: Mayo Clinic experiences with a hitherto unnamed disease. Mayo Clin Proc 55: 434-438, 1980.

8. Matteoni CA, Younossi ZM, Gramlich T, et al: Nonalcoholic fatty liver disease: a spectrum of clinical and pathological severity. Gastroenterology 116: 1413-1419, 1999.

9. Nakamuta M, Kohjima M, Morizono S, et al: Evaluation of fatty acid metabolism-related gene expression in nonalcoholic fatty liver disease. Int J Mol Med 16: 631-635, 2005.

10. Kohjima M, Enjoji M, Higuchi N, et al: Re-evaluation of fatty acid metabolism-related gene expression in nonalcoholic fatty liver disease. Int J Mol Med 20: 351-358, 2007.

11. Shimano H: Sterol regulatory element-binding proteins (SREBPs): transcriptional regulators of lipid synthetic genes. Prog Lipid Res 40: 439-452, 2001.

12. Horton JD, Goldstein JL and Brown MS: SREBPs: activators of the complete program of cholesterol and fatty acid synthesis in the liver. J Clin Invest 109: 1125-1131, 2002.

13. Osborne TF: Sterol regulatory element-binding proteins (SREBPs): key regulators of nutritional homeostasis and insulin action. J Biol Chem 275: 32379-32382, 2000.

14. Matsuzaka T, Shimano H, Yahagi N, et al: Insulin-independent induction of sterol regulatory element-binding protein-1c expression in the livers of streptozotocin-treated mice. Diabetes 53: 560-569, 2004.

15. Kerouz NJ, Horsch D, Pons S and Kahn CR: Differential regulation of insulin receptor substrates-1 and -2 (IRS-1 and IRS-2) and phosphatidylinositol 3-kinase isoforms in liver and muscle of the obese diabetic (ob/ob) mouse. J Clin Invest 100: 3164-3172, 1997

16. Wolfrum C, Asilmaz E, Luca E, et al: Foxa2 regulates lipid metabolism and ketogenesis in the liver during fasting and in diabetes. Nature 432: 1027-1032, 2004.

17. Long YC and Zierath JR: AMP-activated protein kinase signaling in metabolic regulation. J Clin Invest 116: 1776-1783, 2006.

18. Hardie DG: Minireview: the AMP-activated protein kinase cascade: the key sensor of cellular energy status. Endocrinology 144: 5179-5183, 2003.

19. Zhou G, Myers R, Li Y, et al: Role of AMP-activated protein kinase in mechanism of metformin action. J Clin Invest 108: 1167-1174, 2001.

20. Woods A, Azzout-Marniche D, Foretz M, et al: Characterization of the role of AMP-activated protein kinase in the regulation of glucose-activated gene expression using constitutively active and dominant negative forms of the kinase. Mol Cell Biol 20: 6704-6711, 2000.

21. Taniguchi CM, Ueki K and Kahn R: Complementary roles of IRS-1 and IRS-2 in the hepatic regulation of metabolism. J Clin Invest 115: 718-727, 2005.

22. Ide T, Shimano H, Yahagi N, et al: SREBPs suppress IRS-2mediated insulin signalling in the liver. Nat Cell Biol 6: 351-357, 2004.

23. Hardie DG, Carling D and Carlson M: The AMP-activated/ SNF1 protein kinase subfamily: metabolic sensors of the eukaryotic cell? Annu Rev Biochem 67: 821-855, 1998.

24. Yamauchi T, Kamon J, Minokoshi Y, et al: Adiponectin stimulates glucose utilization and fatty-acid oxidation by activating AMP-activated protein kinase. Nat Med 8: 1288-1295, 2002.

25. Hui JM, Hodge A, Farrell GC, et al: Beyond insulin resistance in NASH: TNF-alpha or adiponectin? Hepatology 40: 46-54, 2004.

26. Zelcer $\mathrm{N}$ and Tontonoz $\mathrm{P}$ : Liver $\mathrm{X}$ receptors as integrators of metabolic and inflammatory signaling. J Clin Invest 116: 607-614, 2006. 